\title{
Stability analysis of an SIR model with immunity and modified transmission function
}

\author{
Nidhi Nirwani *, V.H.Badshah, R.Khandelwal \\ School of Studies in Mathematics Vikram University, Ujjain (M.P.) India \\ *Corresponding author E-mail: nd.mathematics2009@gmail.com
}

Copyright $\odot 2015$ Nidhi Nirwani et al. This is an open access article distributed under the Creative Commons Attribution License, which permits unrestricted use, distribution, and reproduction in any medium, provided the original work is properly cited.

\begin{abstract}
This paper examines an SIR epidemic model with a non-monotonic incidence rate. We analyzed the model by considering after infection, only a fraction of transmitted part is shifted to infectious and remaining part gets recovered without becoming infectious. We also analyze the dynamical behavior of the model and derive the stability conditions for the disease-free and the endemic equilibrium. We have found a threshold condition, in terms of basic reproduction number $R_{0}$ which is, less than one, the disease free equilibrium is globally attractive and if more than one, the endemic equilibrium exists and is globally stable. We illustrate theoretical results by carrying numerical simulation.
\end{abstract}

Keywords: Basic Reproduction Number; Disease Frees Equilibrium; Endemic Equilibrium; Epidemiology; Non-Monotonic Incidence; SIR Model; Stability.

\section{Introduction}

The concealed and apparently unpredictable nature of infectious diseases has been a source of fear and superstition since the first ages of human civilization. The asymptotic behavior of solution of an infectious disease transmission model depends not only on epidemiological formation, but also on the demographic process incorporated into the model. Many authors have proposed various kinds of epidemic models to understand the mechanism of disease transmission. Anderson R M [1] proposed transmission and control phenomena of infectious diseases. Diekmann O. Heesterbeek [3] proposed and analyzed the evidence of the increasing diversification of infectious diseases. Several studies are there for the treatment of epidemics with different kind of incidence rates which measures the transfer rate of susceptible to get infected $[4,5,6,9,11,15]$. Thus the incidence is number of new infectious per day or per other time unit. We have several different incidence rates which have been proposed by many researchers in epidemic model. Capasso and Serio [10] introduced a saturated incidence rate $g(I) S$ into epidemic models. Where $g(I)$ tends to a saturation level when $I$ gets large.

Nonlinear incidence rates of form $\beta I^{p} S^{q}$ were investigated by Lui et. al.[12,13]. A very general form of non-linear incidence rate was considered by Derrick and Driessche [14].One of the most fundamental quantities used by epidemiologists is certainty the basic reproduction number consider and analyzed by Anderson R M, May R M[2].In this paper the result is written in terms of basic reproduction number and stability of the equilibriums are investigated.

\section{The mathematical model}

The model we analyze in this paper is considered within the framework of the following nonlinear ordinary differential equations.

$\frac{d S}{d t}=\propto-\beta S-\frac{(1+a I) \mathrm{k} I S}{\left(1+b I^{2}\right)}+\gamma R$ 
$\frac{d I}{d t}=\frac{(1+a I) \rho k I S}{\left(1+b I^{2}\right)}-(\beta+\mu) I$

$$
\frac{d R}{d t}=\mu I-(\beta+\gamma) R+\frac{(1+a I)(1-\rho) \mathrm{k} I S}{\left(1+b I^{2}\right)}
$$

Table 1: Variables and Parameters in the Model

\begin{tabular}{cl}
\hline Symbol & Description \\
\hline$S(t)$ & Number of Susceptible individuals at time t \\
$I(t)$ & Number of Infective individuals at time t \\
$R(t)$ & Number of Recovered individuals at time t \\
$\alpha$ & The recruitment rate of the Population \\
$\beta$ & The natural death rate of the population \\
$\rho$ & A positive constant where $\rho \leq 1$. \\
$\mu$ & The natural recovery rate of the infective Individuals \\
$\gamma$ & The rate at which recovered Individuals lose immunity \\
$k$ & The proportionality constant \\
$a$ & The parameter which measure the affects of medical infrastructure \\
$b$ & The parameter which measure awareness of people through education.
\end{tabular}

Existence of Equilibria

For the system (1), for any values of parameters, it always has a disease free- equilibrium.

$\mathrm{E}_{0}=\left(\frac{\alpha}{\beta, 0,0}\right)$

Define the basic reproduction number as follows:

$\mathrm{R}_{0}=\frac{\rho \alpha \mathrm{k}}{\beta(\beta+\mu)}$

Then we have the following:

\section{Theorem 2.1:}

1) If $R_{0}<1$, then there is no positive equilibrium.

2) If $R_{0}>1$, then there is a unique positive equilibrium $E^{*}=\left(S^{*}, I^{*}, R^{*}\right)$ called the "endemic equilibrium". Given by

$S^{*}=\frac{\left(1+b I^{*^{2}}\right)(\beta+\mu)}{\rho K\left(1+\alpha I^{*}\right)}$

$I^{*}=\frac{-\Delta+\sqrt{\Delta^{2}-4 \beta(\beta+\mu)[\beta b(\beta+\mu)(\beta+\gamma)+\rho K a \beta(\beta+\gamma+\mu)]\left(1-R_{0}\right)}}{2[\beta b(\beta+\mu)(\beta+\gamma)+\rho K a \beta(\beta+\gamma+\mu]}$

$R^{*}=\frac{1}{(\beta+\gamma)}\left[\mu I^{*}+\frac{(1-\rho)\left(1+a I^{*}\right) k I^{*} S^{*}}{\left(1+b I^{*^{2}}\right)}\right]$

$\Delta=\rho K(\beta+\mu)(\beta+\gamma)-\alpha \rho K a(\beta+\gamma)-\rho k \gamma \mu$

Now we check the dynamical behavior of disease free equilibrium point $E_{0}$ and endemic equilibrium point $E^{*}$. The variation matrix of $E_{0}$ is

$V_{0}=\left[\begin{array}{ccc}-\beta & -k \alpha / \beta & \gamma \\ 0 & \rho k \alpha / \beta-(\beta+\mu) & 0 \\ 0 & \mu+(1-\rho) k \alpha / \beta & -(\beta+\gamma)\end{array}\right]$

Its characteristics equation is given by 
$(-\beta-\lambda)(\rho k \alpha / \beta-\beta-\mu-\lambda)(-d-\gamma-\lambda)=0$

Clearly, $E_{0}$ is stable is $\rho k \alpha / \beta<(\beta+\mu)$

$$
V^{*}=\left[\begin{array}{ccc}
-\beta-\left(1+a I^{*}\right) k I^{*} /\left(1+b I^{*^{2}}\right) & -k S^{*}\left(1+b I^{*^{2}}+2 a I^{*}\right) /\left(1+b I^{*^{2}}\right)^{2} & \gamma \\
\left(1+a I^{*}\right) \rho k I^{*} /\left(1+b I^{*^{2}}\right) & \rho k S^{*}\left(1+b I^{*^{2}}+2 a I^{*}\right) /\left(1+b I^{*^{2}}\right)^{2}-(\beta+\mu) & 0 \\
\left(1+a I^{*}\right)(1-\rho) k I^{*} /\left(1+b I^{*^{2}}\right) & \mu+(1-\rho)\left(1+b I^{*^{2}}+2 a I^{*}\right) k S^{*} /\left(1+b I^{*^{2}}\right)^{2} & -(\beta+\gamma)
\end{array}\right]
$$

Its characteristic equation is given by

$$
\begin{aligned}
& \lambda^{3}+a_{1} \lambda^{2}+a_{2} \lambda+a_{3}=0 \\
& a_{1}=\left(3 \beta+\mu+\gamma+\frac{\left(1+a I^{*}\right) k I^{*}}{\left(1+b I^{*^{2}}\right)}-\frac{\rho K S^{*}\left(1+b I^{*^{2}}+2 a I^{*}\right)}{\left(1+b I^{*^{2}}\right)^{2}}\right) \\
& a_{2}=\left(\beta+\frac{\left(1+a I^{*}\right) k I^{*}}{\left(1+b I^{*^{2}}\right)}\right)\left(2 \beta+\mu+\gamma-\frac{\rho K S^{*}\left(1+b I^{*^{2}}+2 a I^{*}\right)}{\left(1+b I^{*^{2}}\right)^{2}}\right)+(\beta+\gamma)\left(\beta+\mu-\frac{\rho K S^{*}\left(1+b I^{*^{2}}+2 a I^{*}\right)}{\left(1+b I^{*^{2}}\right)^{2}}\right) \\
& +\frac{\rho K^{2} S^{*} I^{*}\left(1+b I^{*^{2}}+2 a I^{*}\right)\left(1+a I^{*}\right)}{\left(1+b I^{*^{2}}\right)^{3}}-\frac{\lambda(1-\rho) k I^{*}\left(1+a I^{*}\right)}{\left(1+b I^{*^{2}}\right)}
\end{aligned}
$$

Here $a_{1}>0, a_{2}>0>$ and $a_{3}>0$ provided

$$
\begin{aligned}
& a_{3}=\left(\beta+\frac{k I^{*}\left(1+a I^{*}\right)}{\left(1+b I^{*^{2}}\right)}\right)\left(\beta+\mu-\frac{\rho k S^{*}\left(1+b I^{*^{2}}+2 a I^{*}\right)}{\left(1+b I^{*^{2}}\right)^{2}}\right)(\beta+\gamma)-\frac{\gamma \rho \mu k I^{*}\left(1+a I^{*}\right)}{\left(1+b I^{*^{2}}\right)} \\
& -\frac{\lambda(1-\rho) k I^{*}\left(1+a I^{*}\right)(\beta+\mu)}{\left(1+b I^{*^{2}}\right)}+\frac{\rho k^{2} S^{*} I^{*}\left(1-b I^{*^{2}}+2 a I^{*}\right)\left(1+a I^{*}\right)(\beta+\mu)}{\left(1+b I^{*^{2}}\right)^{3}} \\
& \frac{\rho k^{2} S^{*} I^{*}\left(1-b I^{*^{2}}+2 a I^{*}\right)}{\left(1+b I^{*^{2}}\right)^{2}}<(\beta+\mu)
\end{aligned}
$$

Performing simple calculations it can be easily be verified that $a_{1}, a_{2}>a_{3}$ under the above conditions.

Thus by Routh Hurwitz criterion, all Eigen values of (3) will have negative real part. Hence $E^{*}\left(S^{*}, I^{*}, R^{*}\right)$ is asymptotically stable.

\section{Global analysis}

In this section, we study the properties of the equilibriums and derive the stability condition for the disease- free and the endemic equilibrium of model (1).

Lemma 3.1: The plane $S+I+R=\frac{\alpha}{\beta}$ is an invariant manifold of system (1) which is attracting in the first octant.

Proof: Summing up the three equations in (1) and denoting

$N(t)=S(t)+I(t)+R(t)$, we have

$\frac{d N}{d t}=\alpha-\beta N$

For the equilibrium point, set $\alpha-\beta N=0$ 
From the above equation, it is clear that

$N(t)=\frac{\alpha}{\beta}$

Is one solution of (4) and for any $N\left(t_{0}\right) \geq 0$, the general solution of equation (4) is

$N(t)=\frac{1}{\beta}\left[\alpha-\left(\alpha-\beta N\left(t_{0}\right) e^{-\beta\left(t-t_{0}\right)}\right]\right.$

Also,

$\lim _{n \rightarrow \infty} N(t)=\frac{\alpha}{\beta}$

This completes the proof.

Clearly limit set of a system (1) is on the plane.

$S+I+R=\frac{\alpha}{\beta}$

Thus we focus on the reduced system.

$\frac{d I}{d t}=\frac{k I \rho(1+a I)}{1+b I^{2}}\left(\frac{\alpha}{\beta}-I-R\right)-(\beta+\mu) I \equiv P(I, R)$

$\frac{d R}{d t}=\mu I-(\beta+\gamma) R+\frac{k I(1+a I)(1-\rho)}{1+b I^{2}}\left(\frac{\alpha}{\beta}-I-R\right) \equiv Q(I, R)$

Theorem3.2: System (5) does not have nontrivial periodic orbits.

Proof: Consider system (5) for $I>0$ and $>0$. Take Dulac function [8] as

$D(I, R)=\frac{\left(1+b I^{2}\right)}{(1+a I) \rho K I}$

Then we have

$\frac{\partial(D P)}{\partial I}+\frac{\partial(D Q)}{\partial R}=-1-\frac{(\beta+\mu)}{\rho k}\left[\frac{2 b I+a b I^{2}-a}{(1+a I)^{2}}\right]-\frac{(\beta+\gamma)\left(1+b I^{2}\right)}{\rho k I(1+a I)}-\frac{(1+\rho)}{\rho}$

$=-1-\frac{(1+\rho)}{\rho}-\frac{1}{\rho k I\left(1+a I^{2}\right)}\left[a b(2 \beta+\mu+\gamma) I^{2}+(3 \beta+2 \mu+\gamma) b I^{2}+(\gamma-\mu) a I+(\beta+\gamma)\right]$

Thus the expression (6) is negative for $\gamma \geq \mu$. Hence, the conclusion follows.

In order to study the properties of the disease-free equilibrium $E_{0}$ and the endemic equilibrium $E^{*}$, we rescale (5) by taking

$x=\frac{k}{(\beta+\gamma)} I, y=\frac{k}{(\beta+\gamma)} R, \tau=(\beta+\gamma) t$

Then we obtain,

$\frac{d x}{d \tau}=\frac{x(1+p x) B}{\left(1+q x^{2}\right)}(A-x-y)-m x$

$\frac{d y}{d \tau}=r x-y+\frac{(1+p x)}{\left(1+q x^{2}\right)}(1-B) x(A-x-y)$ 
Where

$A=\frac{\alpha k}{\beta(\beta+\gamma)}, p=\frac{a(\beta+\gamma)}{k}, q=\frac{b(\beta+\gamma)^{2}}{k^{2}}, B=\rho, m=\frac{\beta+\mu}{\beta+\gamma}$,

$r=\frac{\mu}{(\beta+\gamma)}$

The trivial equilibrium $(0,0)$ of the system (7) is the disease-free equilibrium $E_{0}$ of the model (1) and the unique positive equilibrium $\left(x^{*}, y^{*}\right)$ of a system (7) is the endemic equilibrium $E^{*}$ of a model (1) where

$x^{*}=\frac{B y}{m+B(r-m)}, y^{*}=\frac{[m+B(r-m)] x^{*}}{B}$

Which is positive if $r-m>0$ or $r>m$

And $x^{*}$ is the positive solution of the following quadratic equation

$a_{4} x^{2}+a_{5} x+a_{6}=0$

Where

$a_{4}=m q+B p+p[m+B(r-m)]$

$a_{5}=B+[m+B(r-m)]-A B p$

$a_{6}=A B-m$

Obviously, equation (8) has a positive root if $a_{5}<0, a_{5}{ }^{2}-4 a_{4} a_{6}>0$. Obviously Thus, the equilibrium points $E^{*}$ exists.

We first determine the stability and topological type of $(0,0)$. The jacobian matrix of a system $(7)$ at $(0,0)$ is.

$M_{0}=\left[\begin{array}{cc}A B-m & 0 \\ r+(1-B) A & -1\end{array}\right]$

If $A B-m=0$, then there exists a small neighborhood of $(0,0)$ such that the dynamic of a system (7) are equivalent to that of

$\frac{d x}{d \tau}=B(A P-1) x^{2}-x y+O\left((x, y)^{3}\right)$

$\frac{d y}{d \tau}=r x-y+\frac{(1+p x)}{\left(1+q x^{2}\right)}(1-B) x(A-x-y)$

Theorem 3.3: The disease-free equilibrium $(0,0)$ of a system (7) is

i) a stable hyperbolic node if $A<\frac{m}{B}$;

ii) a saddle-node if $A=\frac{m}{B}$;

iii) a hyperbolic saddle if $A>\frac{m}{B}$

When $A>\frac{m}{B}$, we discuss the stability and topological type of the endemic equilibrium $\left(x^{*}, y^{*}\right)$ is

The jacobian of the system (7) at $\left(x^{*}, y^{*}\right)$ is

$M_{1}=\left[\begin{array}{cc}B D-m & -\frac{B(1+p x) x}{\left(1+q x^{2}\right)} \\ r+(1-B) D & -1-\frac{(1-B)(1+p x) x}{\left(1+q x^{2}\right)}\end{array}\right]$

$m+\frac{m(1-B)(1+p x) x}{\left(1+q x^{2}\right)}+\frac{r B(1+p x) x}{\left(1+q x^{2}\right)}>B D$

Thus, if the above condition holds then $\left(x^{*}, y^{*}\right)$ is a node or a focus or a centre. 
The trace of matrix M, is given by

$\operatorname{tr}\left(M_{1}\right)=B D-m-1-\frac{(1-B)(1+p x) x}{\left(1+q x^{2}\right)}$

Thus, $\operatorname{tr}\left(M_{1}\right)<0$ if

$m+1+\frac{(1-B)(1+p x) x}{\left(1+q x^{2}\right)}>B D$

Where

$D=\frac{\left(1+q x^{2}\right)(A-x-y)(1+2 p x)-\left(x+p x^{2}\right)\left[1+q x^{2}+(A-x-y) 2 p x\right]}{\left(1+q x^{2}\right)^{2}}$

Thus, if both the conditions (9) and (10) are satisfied, we get a unique equilibrium $\left(\mathrm{x}^{*}, \mathrm{y}^{*}\right)$ of model (7), which is stable.

\section{Numerical results}

Consider following values of parameters

$\alpha=0.9, \beta=0.82, \mathrm{k}=2.11 \mu=0.11, \rho=0.4,0<\rho<1$

Note that at $\rho=0.4$, we get $R_{0}=1$, when $\rho<0.4$ the value of $R_{0}<1$ and when $\rho>0.4$, the value of $R_{0}>1$ and therefore these exists unique equilibrium.

\begin{tabular}{cllllll}
\hline $\boldsymbol{\rho}$ & 0.1 & 0.2 & 0.3 & 0.4 & 0.5 & 0.6 \\
\hline $\boldsymbol{R}_{\mathbf{0}}$ & 0.1899 & 0.4980 & 0.7470 & 1 & 1.2450 & 1.5192 \\
\hline
\end{tabular}

\section{Conclusion}

In this paper we studied global quantative analysis of a realistic SIR model. In terms of the basic reproduction $\mathrm{R}_{0}$ our main result indicate that when $\mathrm{R}_{0}<1$, the disease free equilibrium is globally attractive. When $\mathrm{R}_{0}>1$, the endemic equilibrium exists and is globally stable. Though the basic reproduction number $\mathrm{R}_{0}$ does not depend on $\mathrm{a}$ and $\mathrm{b}$. This implies that the spread of disease decreases as the social or psychological protective measures for the infective increases. This implies for $\rho=1$, the model coincides with that of Khekare et al. [7]

\section{References}

[1] Anderson RM. Transmission dynamics and control of infectious disease agents. In: Anderson RM, May RM, eds.Population Biology of Infectious Diseases. Springer-Verlag, Berlin, 1982, pp. 149-76. http://dx.doi.org/10.1007/978-3-642-68635-1_9.

[2] Anderson RM, May RM, Infectious Diseases of Humans. Dynamics and Control. Oxford University Press, Oxford, 1991.

[3] Diekmann O, Heesterbeek JAP.Mathematical Epidemiology of Infectious Diseases. Model Building, Analysis and Interpretation. Wiley and Sons, Chichester, 2000.

[4] D. Xiao and S. Ruan: Math. Biosci. 208, 2007, 419-429. http://dx.doi.org/10.1016/j.mbs.2006.09.025.

[5] H.W. Hethcote: The Mathematics of Infectious Disease, SIAM Rew. 42, 2000, 599-633. http://dx.doi.org/10.1137/S0036144500371907.

[6] H.W. Hethcote and P. Van Den Driessche: J. math. Boil. 29, 1991, 271-287.

[7] S Khekare et al., Global Dynamics of an Epidemic Model with a Non-monotonic Incidence Rate, IJOSR-JM, $2014,71-77$.

[8] L.Perko,Differential Equations and Dynamical Systems, Texts in Applied Mathematics,Vol. 7,Springer-Verlag,New work, 1991

[9] S. Ruan and W. Wang: J. Differential equations, 188, 2003, 135-163. http://dx.doi.org/10.1016/S0022-0396(02)00089-X.

[10] V. Capasso and G. Serio: Math. Biosci. 42, 1978, 43-61. http://dx.doi.org/10.1016/0025-5564(78)90006-8.

[11] W. M. Liu, S. A. Levin and Y. Iwasa: J. Math. Biol., 1986, 187-204. http://dx.doi.org/10.1007/BF00276956.

[12] W. M. Liu, H. W. Hethcote and S. Levin: J. Math. Biol., 25, 1987, 359-380. http://dx.doi.org/10.1007/BF00277162.

[13] W.M. Liu, P. van den Driessche, Math. Biosci. 128, 1995, 57-69. http://dx.doi.org/10.1016/0025-5564(94)00067-A.

[14] W.R. DERRICK AND P.VAN DEN DRIESSCHE: J MATH. BIOL. 31, 1993, 495-512. http://dx.doi.org/10.1007/BF00173889.

[15] W. Wang: Math.Biosci. 201, 2006, 58-71. http://dx.doi.org/10.1016/j.mbs.2005.12.022. 